\title{
Formation of protoplanets from massive planetesimals in binary systems
}

\author{
YUSUKE TSUKAMOTO \\ Department of Earth and Planetary science, University of Tokyo,7-3-1 Hongo, Bunkyo-ku, \\ Tokyo 113-0033, Japan; tukamoto@margaux.astron.s.u-tokyo.ac.jp \\ and \\ JUNICHIRO MAKINO \\ Division of Theoretical Astronomy, National Astronomical Observatory, 2-2-1 Osawa, \\ Mitaka, Tokyo 181-8588, Japan
}

\begin{abstract}
More than half of stars reside in binary or multiple star systems and many planets have been found in binary systems. From theoretical point of view, however, whether or not the planetary formation proceeds in a binary system is a very complex problem, because secular perturbation from the companion star can easily stir up the eccentricity of the planetesimals and cause high-velocity, destructive collisions between planetesimals. Early stage of planetary formation process in binary systems has been studied by restricted three-body approach with gas drag and it is commonly accepted that accretion of planetesimals can proceed due to orbital phasing by gas drag. However, the gas drag becomes less effective as the planetesimals become massive. Therefore it is still uncertain whether the collision velocity remains small and planetary accretion can proceed, once the planetesimals become massive. We performed $N$-body simulations of planetary formation in binary systems starting from massive planetesimals whose size is about $100-500 \mathrm{~km}$. We found that the eccentricity vectors of planetesimals quickly converge to the forced eccentricity due to the coupling of the perturbation of the companion and the mutual interaction of planetesimals if the initial disk model is sufficiently wide in radial distribution. This convergence decreases the collision velocity and as a result accretion can proceed much in the same way as in isolated systems. The basic processes of the planetary formation, such as runaway growth and oligarchic growth and final configuration of the protoplanets are essentially the same in binary systems and single star systems, at least in the late stage where the effect of gas drag is small.
\end{abstract}


Subject headings: binaries: close — planetary systems: formation - methods: $n$-body simulations

\section{Introduction}

As of March 2007, 215 candidates of extra-solar planets have been found and at least 30 of them are in binary or multiple star systems (Raghavan et al. 2006). Table 1 shows the candidates of the close binary or multiple star system which has the planets. In this table, $\gamma$ Cephei is an example of close binary systems in which we are interested. According to Hatzes et al. (2003), the semi-major axis and eccentricity of the companion star of $\gamma$ Cephei are 18.5 AU, and 0.36, respectively. Planetary formation process in such a close binary system is the main target of this paper. The frequency of planets in binary systems is not significantly different from that for single star systems (Desidera and Barbieri 2007). It is very important to investigate the formation process in binary system because more than half of stars reside in binary or multiple star systems.

Many authors have investigated planetary accretion process using $N$-body simulation (e.g., Aarseth, Lin, and Palmer 1993; Kokubo and Ida 1996, 1998, 2000, 2002). In all of these simulations, the evolution of protoplanetary disks around isolated stars was studied. On the other hand, Quintana et al. $(2002,2006)$ have investigated the planet formation in binary system from protoplanets by $N$-body simulations. They studied the late stage of the planet formation process (from protoplanets to planets).

Marzari and Scholl (2000) and Thébault et al. (2004, 2006) investigated the distribution of the collision velocity between planetesimals with and without gas drag in binary systems by restricted three-body approach. They found that orbital phasing occurred and collision velocity remained small due to the coupling of gas drag effect and secular perturbation, and they concluded that planetary accretion could proceed. As the planetesimals become massive, however, the gas drag becomes ineffective and mutual gravitational effect between planetesimals becomes important. In such a condition, whether the collision velocity remains small is not clear. As stated above, the final stage, from protoplanets to planets, has been studied by $N$-body simulations, but there has been no $N$-body work on the intermediate stage from massive planetesimals whose size is $100 \mathrm{~km}-500 \mathrm{~km}$ to protoplanets. In this paper, we focus on this intermediate stage.

In a binary system, the orbits of planetesimals change due to perturbation from the companion. The most important term of the perturbation in our simulation region is secular perturbation, if the orbit of the companion is eccentric. The effect of secular perturbation 
is that eccentricity vector moves on the "perturbation circle". Thus, if interaction between planetesimals and gas drag are neglected, planetesimals with different semi-major axis move on the perturbation circles on different frequencies and phases, and therefore gain high relative velocity. This is the reason why the collision velocity becomes high in previous works without gas drag or self gravity (Marzari and Scholl 2000; Thébault et al. 2004, 2006). If the collision velocity becomes high when the planetesimals become massive and thus, the effect of gas drag becomes small, accretion process might halt since collisions might be destructive.

It is a very important question whether destructive collisions occur when the gravitational interaction between planetesimals is taken into account because it determines whether the accretion process can continue after gas drag becomes ineffective. Ito and Tanikawa (2001) studied the evolution of the orbital elements of protoplanets under the perturbation of Jupiter and found that the eccentricities of the protoplanets aligned with each other, resulting in the evolution was very similar to that without the influence of Jupiter. This alignment is due to gravitational interaction between the protoplanets. If this kind of alignment also occur for planetesimals in binary system, the collision velocity can become smaller than the value predicted by Marzari and Scholl (2000).

In this paper, we report the results of $N$-body simulations of protoplanet formation from massive $\left(3 \times 10^{23}-1.4 \times 10^{24} \mathrm{~g}\right)$ planetesimals. We start simulations with the planetesimal disk in which all planetesimals have the same mass. The gravitational interaction between planetesimals is included and gas drag is neglected. We summarize theory of secular perturbation and discuss what initial distribution should be used in section 2. The numerical scheme and initial conditions are described in section 3. We also discuss the validity of the "same mass" assumption of planetesimals in section 3. The results of $N$-body simulations are presented in section 4 . In section 5, we sum up.

\section{Theoretical Preparation}

\subsection{Brief description of secular perturbation}

If the mutual interaction of planetesimals and gas drag are negligible, the orbital evolution of a planetesimal in a binary system can be described by restricted three-body approximation, and the time evolution of eccentricity and longitude of pericenter of the planetesimal are given by secular perturbation theory. The secular evolution of the $h$ and $k$ variables of the planetesimal are expressed as (Heppenheimer 1978; Whitmire et al. 1998; Thébault et 
Table 1: Known candidates of close systems

\begin{tabular}{ccccccc}
\hline \hline \multirow{2}{*}{ Name } & Component & $\begin{array}{c}a \sin i \\
(\mathrm{AU})\end{array}$ & $\begin{array}{c}\text { Projected } \\
\text { Separation } \\
(\mathrm{AU})\end{array}$ & $\begin{array}{c}M \sin i \\
\left(M_{J}\right)\end{array}$ & eccentricity & References \\
\hline HD 041004 & $\mathrm{B}$ & $\ldots$ & 22 & $\ldots$ & $\ldots$ & $1,2,3,4$ \\
& $\mathrm{C}$ & 0.016 & $\ldots$ & 18.4 & 0.08 & 3,4 \\
& $\mathrm{~b}$ & 1.31 & $\ldots$ & 2.3 & 0.39 & \\
\hline$\gamma$ Cephei & $\mathrm{B}$ & 20.3 & $\ldots$ & $\ldots$ & 0.39 & $1,5,6,7,8$ \\
& $\mathrm{~b}$ & 2.03 & $\ldots$ & 1.59 & 0.2 & \\
\hline GJ 893 & $\mathrm{B}$ & $\ldots$ & 2248 & $\ldots$ & $\ldots$ & 9,10 \\
& $\mathrm{C}$ & $\ldots$ & 18 & $\ldots$ & $\ldots$ & \\
& $\mathrm{b}$ & 0.3 & $\ldots$ & 2.9 & $\ldots$ & \\
\hline \hline
\end{tabular}

Note. - These parameters are from Raghavan et al. (2006) and its references. Column component lists companions $(\mathrm{B}, \mathrm{C}, \mathrm{D}, \ldots)$ and planets $(\mathrm{b}, \mathrm{c}, \mathrm{d}, \ldots)$.

References. - (1)Eggenberger et al. 2004; (2)See 1896; (3) Zucker et al. 2003; (4) Zucker et al. 2004; (5) Mason et al. 2001; (6) Campbell et al. 1988; (7) Griffin et al. 2002; (8) Hatzes et al. 2003; (9) Zacharias et al. 2004; (10) Wilson 1953

al. 2006)

$$
\begin{aligned}
& h(t)=e_{p} \sin \left(A t+\varpi_{0}\right), \\
& k(t)=e_{p} \cos \left(A t+\varpi_{0}\right)+e_{f},
\end{aligned}
$$

where

$$
\begin{aligned}
& h(t)=e \sin (\varpi), \\
& k(t)=e \cos (\varpi),
\end{aligned}
$$

and $e$ and $\varpi$ are the eccentricity and the longitude of pericenter of the planetesimal. Here, $A, e_{p}, \varpi_{0}$, and $e_{f}$ are constants which depend on the strength of the secular perturbation. We take the $k$ axis as direction of the eccentricity vector of the companion. In other words, the eccentricity vector of the companion is $\left(e_{B}, 0\right)$. The semi-major axis of the companion is 
$a_{B}$ and its mass is $M_{B}$. According to the secular perturbation theory, $e_{f}$ is given by

$$
e_{f}=\frac{5}{4} \frac{a}{a_{B}} \frac{e_{B}}{\left(1-e_{B}^{2}\right)} .
$$

This $e_{f}$ is forced eccentricity induced by the companion star. Here, $a$ is the semi-major axis of the planetesimal. The angular velocity of rotation on $\mathrm{k}-\mathrm{h}$ plane, $A$, is given by

$$
A=\frac{3}{2} \pi \frac{1}{\left(1-e_{B}^{2}\right)^{3 / 2}} m_{B} \frac{a^{3 / 2}}{a_{B}^{3}} .
$$

We use system of units in which the solar mass, $1 \mathrm{AU}$ and 1 year are all unity. Remaining two constants, $\varpi_{0}$ and $e_{p}$, are determined from the initial orbital elements of the planetesimal. We call the vectors $\mathbf{e}=(k, h), \mathbf{e}_{f}=\left(e_{f}, 0\right)$ the eccentricity vector and the forced eccentricity vector, respectively. On $\mathrm{k}-\mathrm{h}$ plane, the eccentricity vector of a planetesimal moves on a circle centered at $\mathbf{e}_{f}$.

\subsection{Initial eccentricity of planetesimals}

We consider two types of initial conditions for planetesimals. In the first one, the initial distribution of eccentricity vectors of planetesimals is centered at the forced eccentricity vector. The distributions of $\mathbf{e}-\mathbf{e}_{f}$ and inclination $i$ are both given by Rayleigh distribution with dispersions $\left\langle\left|\mathbf{e}-\mathbf{e}_{f}\right|^{2}\right\rangle^{1 / 2}=2\left\langle i^{2}\right\rangle^{1 / 2}=0.02$. We call this model "forced" model. In the second model, the distribution of eccentricity vector is centered at the origin of $\mathrm{k}$-h plane. The distributions of eccentricity $e$ and inclination $i$ are also given by Rayleigh distribution with dispersions $\left\langle e^{2}\right\rangle^{1 / 2}=2\left\langle i^{2}\right\rangle^{1 / 2}=0.02$. This is the same distribution as those used in previous works for $\mathrm{N}$-body simulation of planetary formation in isolated systems (Kokubo and Ida 2002). We call this model "circular" model.

We argue that the "forced" model is more suitable for simulation in binary system than the circular model for the following reasons. Consider a narrow region of a planetesimal disk such as the region of $0.95 \mathrm{AU}<a<1.05 \mathrm{AU}$. If the eccentricity vectors of planetesimals in this region do not distribute around the forced eccentricity vector, they will rotate around the position of the forced eccentricity vector. The planetesimals in a narrow region would rotate together because the angular velocity $A$ is similar for planetesimals with similar values of the semi-major axis $a$. In addition, they align due to secular interactions between planetesimals (see, e.g., Ito and Tanikawa 2001), Because of this collective motion, the relative velocity between planetesimals is kept small in this narrow "ring".

However, if we consider a wider region, it would behave as collection of many narrow

rings. If we ignore interactions between the rings, they would rotate on their own angular 
velocities $A$. Since neighboring rings have slightly different values of $A$, they would soon physically collide with each other, resulting in damping of "free" eccentricity. Gravitational interaction between rings would also dump relative difference of eccentricity vectors. In other words, planetesimals in the circular model would first relax to the forced model. We argue that the equilibrium state is more suitable for the initial conditions. This is why we consider "forced" model.

As we mentioned in introduction, we study the late stage of the formation process of protoplanets. The earlier phase have been studied by Marzari and Scholl (2000) and others. Thus, it might seem reasonable to set the initial eccentricity and longitude of pericenter of planetesimals to the equilibrium value of the orbital phasing in Marzari and Scholl (2000) instead of the circular model. However, We found it is a bit difficult to use their results, because they adopt circular gas disk model. This circular gas disk in a binary system with eccentric companion seems a bit unnatural. Due to the perturbation from the companion star, the gas disk may be twisted. We could not find previous works which directly studied the equilibrium state of gas disk under the secular perturbation of companion, but recent work by Papaloizou (2005) seems to imply that eccentric disk can be long-lived even if there is no companion. So it seems likely that gas disk is not circular when eccentric companion exists. This may change gas drag effect to the planetesimals and might affects the direction of the orbital phasing. This is the reason why we do not use the Marzari's results. Even if the orbital elements of them is correct, our circular model is very close to their results and it would soon relax to the "forced" model. So we expect that it makes no significant change to our results. Clearly, more studies on the dynamics of gas disk is necessary.

\subsection{Collision velocity}

As noted in section 2.1, we adopt the Rayleigh distribution with dispersions $\left\langle e^{2}\right\rangle^{1 / 2}=$ $2\left\langle i^{2}\right\rangle^{1 / 2}=0.02$ for the " circular" model (Ida and Makino 1992), and $\left\langle\left|\mathbf{e}-\mathbf{e}_{f}\right|^{2}\right\rangle^{1 / 2}=2\left\langle i^{2}\right\rangle^{1 / 2}=$ 0.02 for the "forced" model (see Table 2 ). These conditions imply collision velocity is initially $v_{c o l} \simeq 500-1000 \mathrm{~m} \mathrm{~s}^{-1}$ at $1 \mathrm{AU}$ for the initial distribution. When the effect of gas drag is taken into account, the equilibrium eccentricity and inclination are estimated as $\left\langle e^{2}\right\rangle^{1 / 2}=2\left\langle i^{2}\right\rangle^{1 / 2}=0.0042$ (Kokubo and Ida 2000). This value is probably more reasonable. In this paper, however, we use the value 0.02 because we neglect gas drag force in our calculation and the value of the eccentricity and inclination will soon relax to the value of gas-free case. Our focus is not on the quantitative analysis of the collision velocity but on the qualitative analysis of the coupling of secular perturbation and gravitational interactions between planetesimals. To determine the realistic value of the collision velocity, we should 
include gas drag force in our calculation.

\section{Method of Calculation}

\subsection{Initial conditions}

The model parameters for the companion star are summarized in table 2. Mass of the primary star is $1 M_{\odot}$ for all models. We adopt three models for companion. Model alpha corresponds to $\alpha$ Cen system. The mass ratio is chosen to be the same as that of $\alpha$ Cen system, (1.1:0.9). For the other two models, we use a somewhat smaller semi-major axis than those of observed binary systems in which the planets are found. We choose this value to study the case in which secular perturbation is strong. For circular models, we use an axisymmetric surface mass density distribution for planetesimal disk whose surface mass density is given by

$$
\Sigma_{\text {solid }}=\Sigma_{0}\left(\frac{a}{1 \mathrm{AU}}\right)^{-3 / 2} \mathrm{~g} \mathrm{~cm}^{-2},
$$

where $a$ is distance from the primary star, and $\Sigma_{0}$ is the reference surface density at $1 \mathrm{AU}$ (We adopt $\Sigma_{0}=10 \mathrm{~g} \mathrm{~cm}^{-2}$ ). Table 3 shows model parameters. Here, $N$ is the number of planetesimals. For radial distribution, we use two disk models, "wide disk" models (models 0 - 5) and "narrow disk" models (models 6 - 8). In the "wide disk" models, we set inner and outer cutoff radii to be $0.5 \mathrm{AU}$ and 1.5 AU, respectively. In the "narrow disk" models, inner and outer cutoff radii are $0.95 \mathrm{AU}$ and $1.05 \mathrm{AU}$, respectively. Planetesimals have equal mass in all models. The number of planetesimals is 10000 in the wide disk models. In two narrow disk models (model 6,7), Number of planetesimals is 5000. In one of narrow disk models (model 8), the number of planetesimals is 975. This is a "cutoff" model which has the planetesimals of the same mass as in the wide disk models. The density of planetesimals is $2 \mathrm{~g} \mathrm{~cm}^{-3}$. We increase their radii by a factor 5 to accelerate accretion process (Kokubo and Ida 1996). At first, all planetesimals have same mass $\left(\simeq 1.44 \times 10^{24} \mathrm{~g}\right.$ in models $0-5$ and 8 and $2.88 \times 10^{23} \mathrm{~g}$ in model 6 and 7 ).

One critical question is if our "same mass" setup can be really regarded as description of the later stage of planetary formation. Recent theoretical and numerical works of planetary accretion (for example Inaba et al. 2001, Rafikov 2003) seem to suggest that the "orderly" phase of growth does not exist, and runaway growth starts from much smaller mass than that of our setup. Our setup, therefore, is not realistic. However, statistical calculation

by Inaba et al. (2001) has shown that once massive planetesimals $\left(M \simeq 10^{23} \mathrm{~g}\right)$ formed, planetesimals with mass less than $10^{21} \mathrm{~g}$ become dynamically unimportant. So even though 
our initial condition is oversimplified, it might still give qualitatively valid description of the later phase.

Two planetesimals are considered to collide when their distance becomes less than the sum of their radii. We assume the perfect accretion under which planetesimals always accrete when they collide. Whether or not this assumption is good depends on the distribution of the collision velocities. The escape velocity of our planetesimals is $200-600 \mathrm{~m} \mathrm{~s}^{-1}$ and is of the same order as the initial collision velocity. Furthermore, if gas effect is included, the eccentricity dispersion might be less than our estimate and the collision velocity becomes smaller. So we believe the assumption of perfect accretion is valid.

As stated above, we increase the radii of planetesimals $f$-fold (here, $f=5$ ) to save calculation time. This acceleration of accretion may affect time evolution of planetesimals, especially the time evolution of orbital elements on $\mathrm{k}$-h plane because the increase of radii changes timescale of accretion but does not affect secular perturbation of companion star.

Table 2: Model parameters for the companion star

\begin{tabular}{cccc}
\hline \hline Name & Mass & $\begin{array}{c}\text { semi-major axis } \\
(\mathrm{AU})\end{array}$ & eccentricity \\
\hline nocomp & 0 & $\ldots$. & $\ldots$. \\
e25 & $1 M_{\odot}$ & 16 & 0.25 \\
e50 & $1 M_{\odot}$ & 16 & 0.5 \\
alpha & $0.82 M_{\odot}$ & 23.4 & 0.52 \\
\hline
\end{tabular}

\subsection{Integration scheme}

We use the fourth-order Hermite scheme (Makino and Aarseth 1992) with hierarchical timesteps (Makino 1991) improved for planetary systems (Kokubo et al. 1998 ) for numerical integration of planetesimals and companion star. The equation of motion for planetesimals is given by

$$
\mathbf{a}_{i}=-\sum_{i \neq j} G m_{j} \frac{\mathbf{r}_{\mathbf{i j}}}{r_{i j}^{3}}-G\left(m_{i}+M_{p}\right) \frac{\mathbf{r}_{\mathbf{i p}}}{r_{i p}^{3}}-G M_{c}\left(\frac{\mathbf{r}_{\mathbf{i c}}}{r_{i c}^{3}}+\frac{\mathbf{r}_{\mathbf{p c}}}{r_{p c}^{3}}\right),
$$

where $M_{p}, M_{c}, r_{i p}$, and $r_{i c}$ are mass of the primary star, mass of companion star, position of planetesimal relative to the primary star and that relative to the companion star. We use 
Table 3: Initial conditions

\begin{tabular}{ccccc}
\hline \hline Model & $N$ & $\begin{array}{c}\text { Width of disk } \\
(\mathrm{AU})\end{array}$ & companion & initial orbit \\
\hline 0 & 10000 & $0.5-1.5$ & nocomp & $\ldots$ \\
1 & 10000 & $0.5-1.5$ & $\mathrm{e} 25$ & forced \\
2 & 10000 & $0.5-1.5$ & $\mathrm{e} 50$ & forced \\
3 & 10000 & $0.5-1.5$ & $\mathrm{e} 25$ & circular \\
4 & 10000 & $0.5-1.5$ & $\mathrm{e} 50$ & circular \\
5 & 10000 & $0.5-1.5$ & alpha & circular \\
6 & 5000 & $0.95-1.05$ & $\mathrm{e} 25$ & circular \\
7 & 5000 & $0.95-1.05$ & alpha & circular \\
8 & 975 & $0.95-1.05$ & $\mathrm{e} 25$ & circular \\
\hline
\end{tabular}

position of the primary star as origin of the coordinate for planetesimals. The motion of the primary star due to the gravitational forces of planetesimals is neglected.

Most expensive part of the numerical integration is calculation of mutual gravitational interaction between planetesimals. We use GRAPE-6 (Makino et al. 2003) and GRAPE-6A (Fukushige et al. 2005) to calculate the gravitational interaction between planetesimals. We also integrate orbit of the companion star using the fourth-order Hermite scheme. For both the planetesimals and the companion stars, we use the standard timestep criterion (Aarseth 1985)

$$
\Delta t=\sqrt{\eta \frac{|a|\left|a^{(2)}\right|+|\dot{a}|^{2}}{|\dot{a}|\left|a^{(3)}\right|+\left|a^{(2)}\right|^{2}}} .
$$

Since the orbital period of the companion is long, the accuracy of its orbit is more than enough.

\section{Results}

\subsection{Planetary accretion in binary systems}

Figure 1 shows the time evolution of planetesimals of model 1 (left) and model 0 (right) on the a-e plane. We integrate the system for $5 \times 10^{5}$ years. We can see that protoplanets grow in a very similar way in these two models. This behavior is essentially the same for all models. 
Figure 2 shows cumulative mass distribution of planetesimals of the region $0.9 \mathrm{AU}<a<$ 1.1AU. The time evolutions of all of these models are very similar. In all cases, the mass distributions first relax to the power-law distribution with power index $d \log n_{c} / d \log m \simeq$ -1.5 (top panels) where $n_{c}$ is the cumulative number of planetesimals. This power-law distribution is characteristic of the runaway growth (Makino et al. 1998, Kokubo and Ida 2000).

As time goes on, the mass distributions become bimodal: planetesimals with mass $M \simeq 1-10 \times 10^{24} \mathrm{~g}$ and large protoplanets with mass $M \simeq 10^{27} \mathrm{~g}$. This bimodal mass distribution is the result of the runaway and the oligarchic growth of protoplanets (Kokubo and Ida 1996,1998). The time evolution of the mass distribution of binary systems is almost the same as that in single star systems. It means that secular perturbation does not change the process of the protoplanet formation. Runaway growth and oligarchic growth also occur in binary systems in a similar way as in the single star system.

In figure 3- 6, we show the evolution of the mass of most massive planetesimals (3) and 44) and average mass of planetesimals (5 and 6). We can see that the evolution is rather similar and there is no systematic tendency due to the presence of the companion.

The evolution of the surface mass density of model 1 ( $e=0.25$, forced) and that of model 0 (no companion) are shown in Figure 7. We found that density profile of planetesimals in a binary system is virtually the same as that in a single star system.

From secular perturbation theory, time evolution of semi-major axis of a planetesimal is given by

$$
\frac{d a}{d t}=\frac{2}{n a} \frac{\partial R}{\partial \epsilon}
$$

where $\epsilon$ and $R$ are mean longitude at epoch and disturbing function, respectively. In practice, variation of $\epsilon$ can usually be neglected since it is a small effect. Thus, mass migration does not occur since secular perturbation hardly change the semi-major axis of planetesimals. Our results are consistent with this theoretical expectation.

\subsection{Time evolution of eccentricity}

The time evolutions of the distribution of the planetesimals on $\mathrm{k}$-h plane of model 1 (forced model of $e=0.25$ ), 3 (circular model of $e=0.25$ ), 8 (cutoff model of $e=0.25$ ), 4, and 5 (circular models) are shown in Figure 8 .

The top three panels show models $1,3,8$, all with an e25 companion. In model 1 (top 
panel), planetesimals are initially distributed around the forced eccentricity, and this does not change in time. In model 3, planetesimals are initially distributed around zero eccentricity, but this initial distribution is replaced by a distribution centered at the forced value. The behavior of planetesimals in model 8 is very different. The distribution after 30,000 years is not centered at the forced value and keeps rotating around the forced eccentricity vector (see figure 9 and description below). This difference between narrow disk models and wide disk models will be discussed in more details in section 4.2.1. The bottom two panels of figure 8 show that the eccentricity vectors of the planetesimals of other circular models with wide distribution also converge to the forced eccentricity vector. We will discuss the time evolution of proper eccentricity in section 4.2.2.

\subsubsection{Difference between wide and narrow models}

We performed simulations of the narrow disk models (models 6, 7,8) to see the difference between the wide and narrow distributions. We use two types of narrow disk models. One has the planetesimals of the same mass as in wide disk models, and thus the number of planetesimals is small $(N=975$, model 8$)$. The other has a large number of smaller planetesimals (see Table 2).

Figure 9 shows the time evolution of eccentricity. In wide disk models, the oscillation of eccentricity is damped quickly due to the convergence to the forced eccentricity vector as shown in figure 8. In narrow disk models, however, the eccentricity liberates around the forced eccentricity and does not converge to the forced eccentricity.

This difference can be understood as follows. As we discussed in section 2, the planetesimals rotate around the forced eccentricity vector on $\mathrm{k}$-h plane and the angular velocity $A$ is a function of semi-major axis of planetesimals. In the case of narrow disk models, the range of $A$ is small, and it is possible that all planetesimals synchronize due to mutual gravitational interaction. Thus in narrow disk models planetesimals move collectively on $\mathrm{k}$-h plane. In the case of the wide disk models, however, such collective motion is not allowed since the range of $A$ is too large. If planetesimals with different values of $a$ (therefore, $A$ ) rotate around the its own values of forced eccentricity on their own timescales, collision is enhanced and eccentricity will be damped to forced values. Thus, oscillation of eccentricity damps quickly in wide models.

We performed a simulation of narrow disk models to see how this behavior is affected by the mass of planetesimals. The bottom panel of Figure 9 shows the time evolution of eccentricity of model 8 . The behavior of model 8 is almost the same as that of model 5 . 
Thus, the number of planetesimals or mass of each planetesimal do not change the result. In narrow disk models, the convergence to the forced eccentricity vector never occur.

\subsubsection{Time evolution of proper eccentricity}

Time evolution of proper eccentricity, (i.e., distance from the forced eccentricity in k$\mathrm{h}$ plane) is shown in figure 10. The proper eccentricity is averaged for planetesimal with semi-major axis between 0.95 AU $<a<1.05 \mathrm{AU}$. In circular models (model 3, 4, and 5 ), the proper eccentricity quickly decreases through collisional damping and gravitational relaxation. This means that the eccentricity vector of the planetesimals converge to the forced eccentricity vector. In forced models (model 1,2) and single star model (model 0), on the other hand, the proper eccentricity does not change significantly in early stage of the simulation (before 40,000 years). The convergence in model 5 ( $\alpha$ Cen model) is slower than that in the other two circular models and before convergence the planetary accretion has almost finished. This is because of the small angular velocity $A$ of $\alpha$ Cen system. However, if we adopt real radii, the convergence would probably occur before the accretion completes. The time evolution of proper eccentricity after the convergence is similar to that in the forced models and the single star model. The proper eccentricity increases in the late stage due to the viscous stirring.

Figure 11 shows time evolution of the mass weighted average of proper eccentricity for the same radial range as in figure 10, In the early stage of the simulation, its behavior is similar to that of the simple average in figure 10. However, in the late stage, the increase is slow because of dynamical friction to seeds of protoplanet. In single star systems, the eccentricity of seeds of protoplanet becomes small (i.e., the eccentricity vectors converge to the origin of $\mathrm{k}$-h plane). On the other hand, in binary systems, the eccentricity vectors converge to the forced eccentricity and the evolution of proper eccentricity is similar to the evolution of eccentricity in the single star system. This means that the orbit of planetesimals does not become circular as in single star system but become eccentric in binary systems and their pericenter aligns to the pericenter of companion star.

The distance between planetesimals on $\mathrm{k}$-h plane determines the collision velocity. Thus, the convergence reduces the collision velocity between planetesimals. In model alpha, for example, the maximum distance between planetesimals could becomes 0.08 by secular oscillation if the mutual gravitational effect is neglected. It corresponds to the collision velocity

of $3000-4000 \mathrm{~m} \mathrm{~s}^{-1}$ at $1 \mathrm{AU}$. Collision with this velocity is destructive even for the mass of the planetesimal of about $1.4 \times 10^{24} \mathrm{~g}$ (its escape velocity is about $600 \mathrm{~m} \mathrm{~s}^{-1}$ ). On the other hand, The collision velocity is reduced to about $500-1000 \mathrm{~m} \mathrm{~s}^{-1}$ with this convergence. As 
we mentioned above, gas drag affects the eccentricity dispersion of the planetesimals (i.e., gas drag can not be negligible in this sense). The simulations in which gas drag and mutual gravitational effects are taken into account is required to determine the precise value of the collision velocity.

\section{Conclusion and Discussion}

We have performed the simulations of formation of protoplanets from massive planetesimals (with radii of $100 \mathrm{~km}-500 \mathrm{~km}$ ) in close binary systems, for which gas drag effect is negligible and the coupling of the secular perturbation and gravitational interaction is important.

We found that eccentricity vectors of planetesimals quickly converge to the forced eccentricity if the initial disk model is sufficiently wide in radius and secular perturbation is sufficiently strong. The eccentricity vectors of planetesimals which have heavier mass than the other planetesimals converge to the forced eccentricity more strongly. This convergence results in orbital phasing and it reduces value of collision velocity to less than the value predicted in studies with restricted three-body approach. Runaway growth and oligarchic growth also occur in binary system much in the same way as isolated star system at least in the late stage of the formation. The final configuration of protoplanets is not different between close binary systems and isolated star systems.

Our simulations, however, have following limitations. (1) We underestimated effect of secular perturbation. The f-fold change of radius increases frequency of collision and accelerates planetary accretion. This acceleration of the formation process causes relative under-estimate of secular perturbation. We plan to perform the simulation with real radii of planetesimals to see if this effect would make any difference. (2) We investigated only three binary systems. More simulations of various binary systems are required to determine quantitative relationship between the convergence of eccentricity vectors and strength of secular perturbation. (3) We neglected effect of gas drag. This is okay for the late stage which we studied. In earlier stage, however, gas drag is clearly important. We plan to calculate the equilibrium state of gas disk under the secular perturbations and perform the simulations which include the gas effect in future works. 


\section{Acknowledgments}

We express our sincere gratitude to Masaki Iwasawa and Keigo Nitadori for useful comments on our $\mathrm{N}$-body code. We also thank Eiichiro Kokubo and Satoshi Inaba for many useful suggestions. We thank the anonymous referee whose critical comments helped us greatly to make the paper clear. This research is partially supported by the Special Coordination Fund for Promoting Science and Technology (GRAPE-DR project), Ministry of Education, Culture, Sports, Science and Technology, Japan.

\section{REFERENCES}

Aarseth, S. j. 1985, in Multiple Time Scales, ed. J. U. Brackhill and B. I. Cohen (Academic Press, New York), p. 377.

Aarseth, S. J., Lin, D. N. C., Palmer, P. L. 1993, ApJ, 403, 351

Campbell, B., Walker, G. A. H., \& Yang, S. 1988, ApJ, 331, 902

Desidera, S., Barbieri, M., 2007, A\&A, 462, 345

Eggenberger, A., Udry, S., \& Mayor, M. 2004, A\&A, 417, 353

Fukushige, T., Makino, J., \& Kawai, A. 2005, PASJ, 57, 1009

Griffin, R. F., Carquillat, J.-M., \& Ginestet, N. 2002, The Observatory, 122, 90

Heppenheimer, T., 1978, A\&A, 65, 421

Hatzes, A.P., Cochran, W.D., Endl, M., et al. 2003, ApJ. 599, 1382

Ito, T., Tanikawa, K. 2001, PASJ, 53, 143

Inaba, S., Tanaka, H., Nakazawa, K., Wetherill, G. W., \& Kokubo, E. 2001, Icarus, 149, 235

Kokubo, E. \& Ida, S. 1996, Icarus, 123, 180

- 1998, Icarus, 131, 171

—. 2000, Icarus, 143, 15

- 2002, ApJ, 581, 666

Kokubo, E., Yoshinaga, K., \& Makino, J. 1998, MNRAS, 297, 1067 
Makino, J. 1991b, PASJ, 43, 859

Makino, J., \& Aarseth, S. J. 1992, PASJ, 44, 141

Makino, J., Fukushige, T., Funato, Y., \& Kokubo, E. 1998, New Astronomy, 3, 411

Makino, J., Fukushige, T., Koga, M., \& Namura, K. 2003, PASJ, 55, 1163

Marzari F., Scholl H., 2000, ApJ, 543, 328

Mason, B. D., Hartkopf, W. I., Holdenried, E. R., \& Rafferty, T. J. 2001, AJ, 121, 3224

Papaloizou, J. C. B. 2005, A\&A, 432, 757

Quintana, E. V., Lissauer, J. J., Chambers, J. E., \& Duncan, M. J. 2002, ApJ, 576, 982

Quintana, E. V., \& Lissauer, J. J. 2006, Icarus, 185, 1

Rafikov, R. R. 2003, AJ, 125, 942

Raghavan, D., Henry, T. J., Mason, B. D., Subasavage, J. P., Jao, W.-C., Beaulieu, T. D., \& Hambly, N. C. 2006, ApJ, 646, 523

See, T. J. J. 1896, AN 142, 43

Thébault, P., Marzari, F., Scholl, H., Turrini, D., Barbieri, M.,2004, A\&A, 427, 1097

Thébault, P., Marzari, F., Scholl, H., 2006, Icarus, 183, 193

Whitmire, D., Matese, J., Criswell, L., 1998, Icarus, 132, 196

Wilson, R. E. 1953, General Catalogue of Stellar Radial Velocities (Washington, DC: Carnegie Inst. Washington)

Zacharias, N., Urban, S. E., Zacharias, M. I., Wycoff, G. L., Hall, D. M., Monet, D. G., \& Rafferty, T. J. 2004, AJ, 127, 3043

Zucker, S., Mazeh, T., Santos, N. C., Udry, S., \& Mayor, M. 2003, A\&A, 404, 775

Zucker, S., Mazeh, T., Santos, N. C., Udry, S., \& Mayor, M. 2004, A\&A, 426, 695 

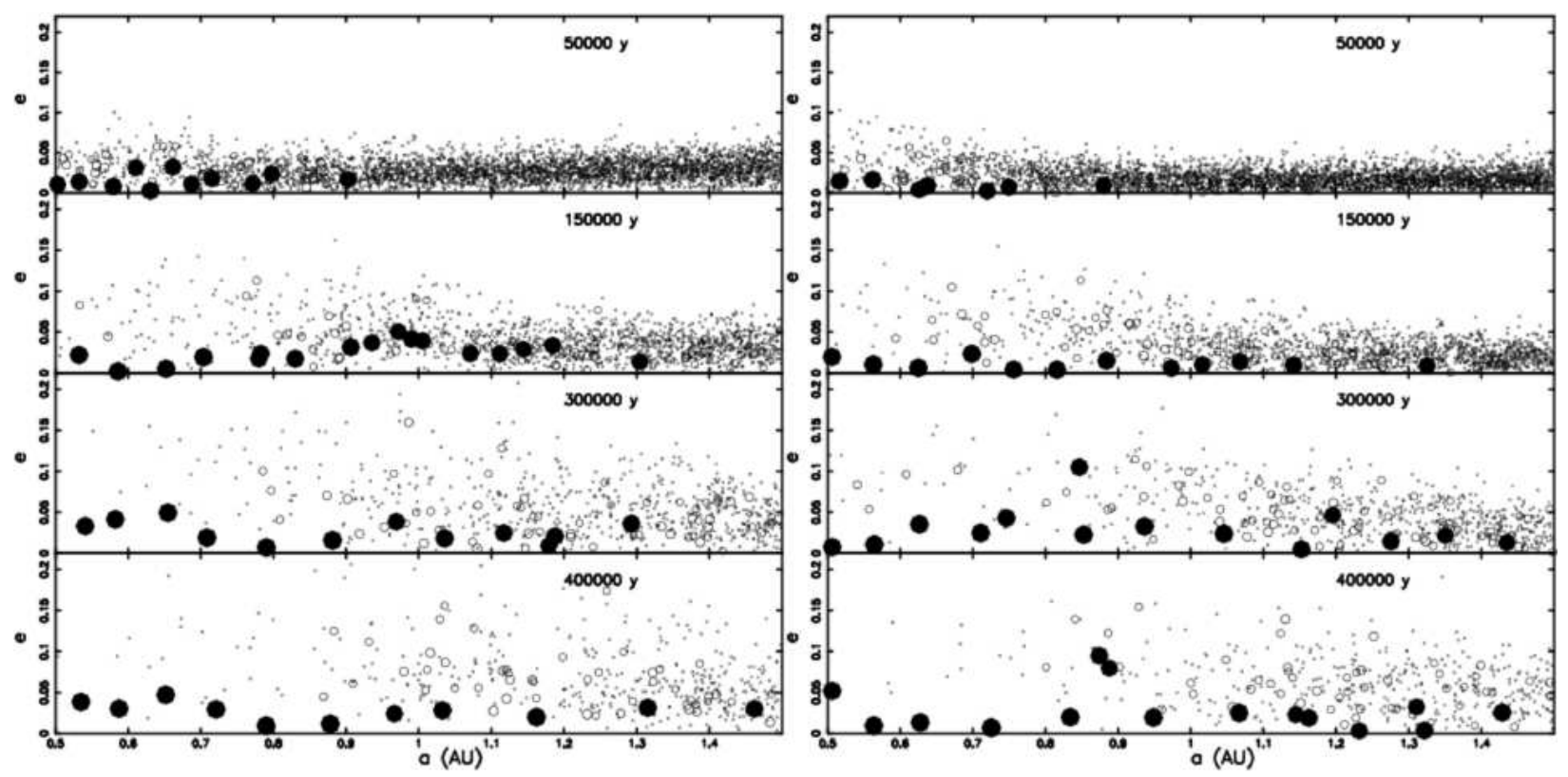

Fig. 1.- Distribution of planetesimals on the $a$-e plane for model 1 (left) and model 0 (right). The circles represent planetesimals. The filled circle represent protoplanets with mass more than 100 times the initial mass. 

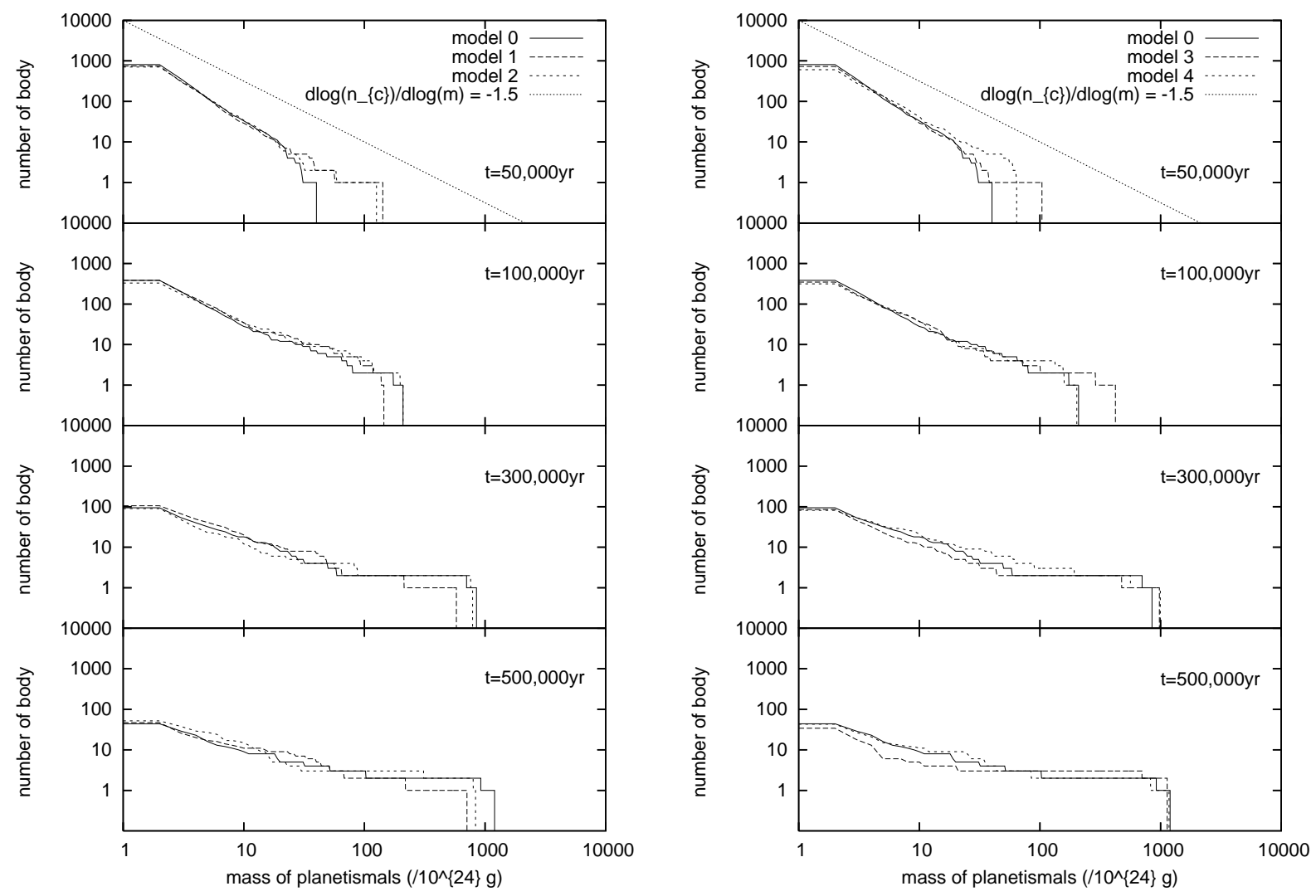

Fig. 2.- Cumulative mass distribution of planetesimals. Left panels show the results of the forced models (model 1,2) and right panels show the results of the circular models (model 3,4). In all panels, the results of isolated model (model 0) are also shown. Times are $5 \times 10^{4}, 1 \times 10^{5}, 3 \times 10^{5}, 5 \times 10^{5}$ years from top to bottom. The dotted lines at the top panels indicate the slope of -1.5 . 


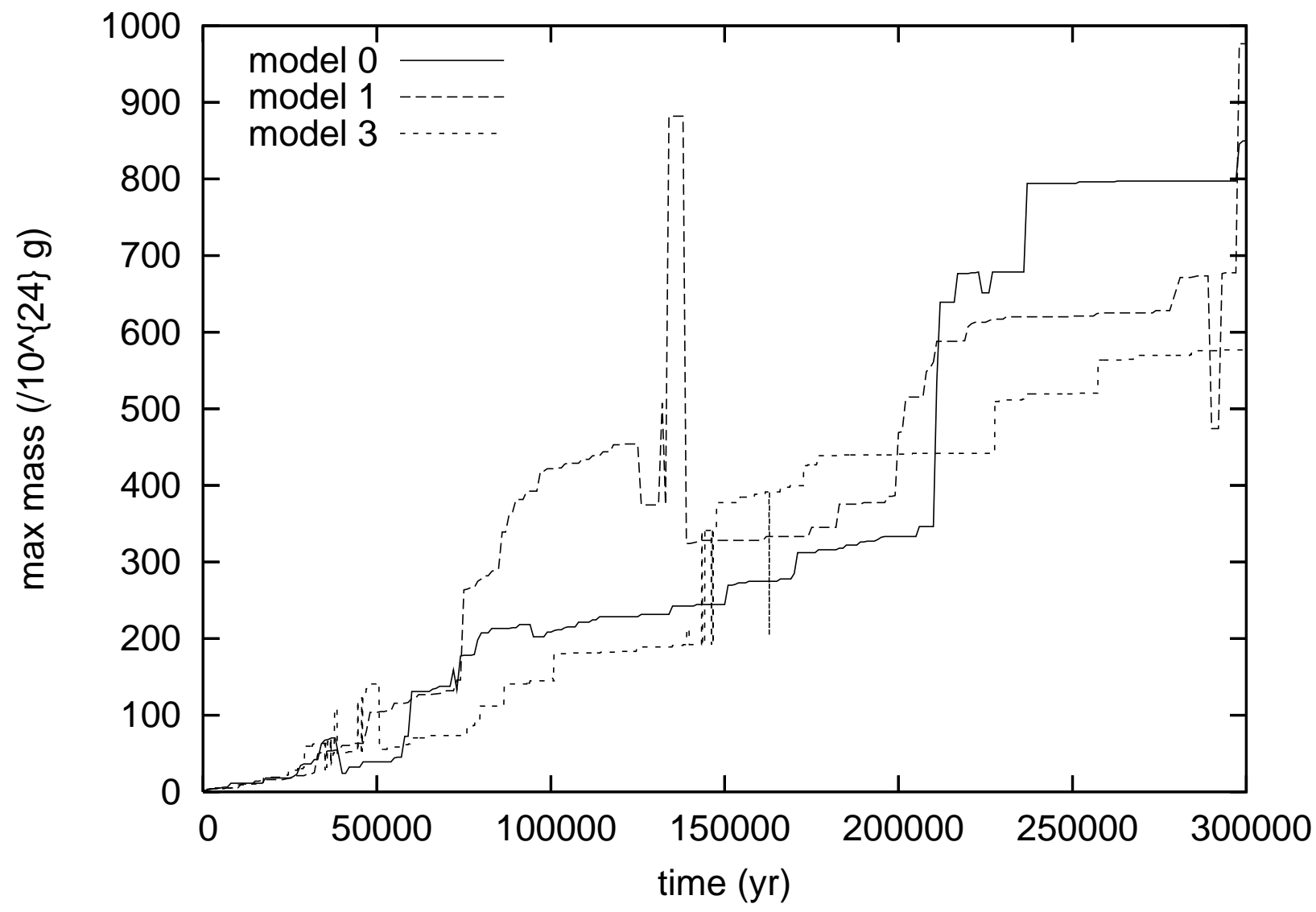

Fig. 3. - The time evolution of maximum mass of planetesimals between $0.9 \mathrm{AU}<a<$ 1.1AU. Solid, dashed, and dotted curves show the result of the model 0,1 , and 3 , respectively. 


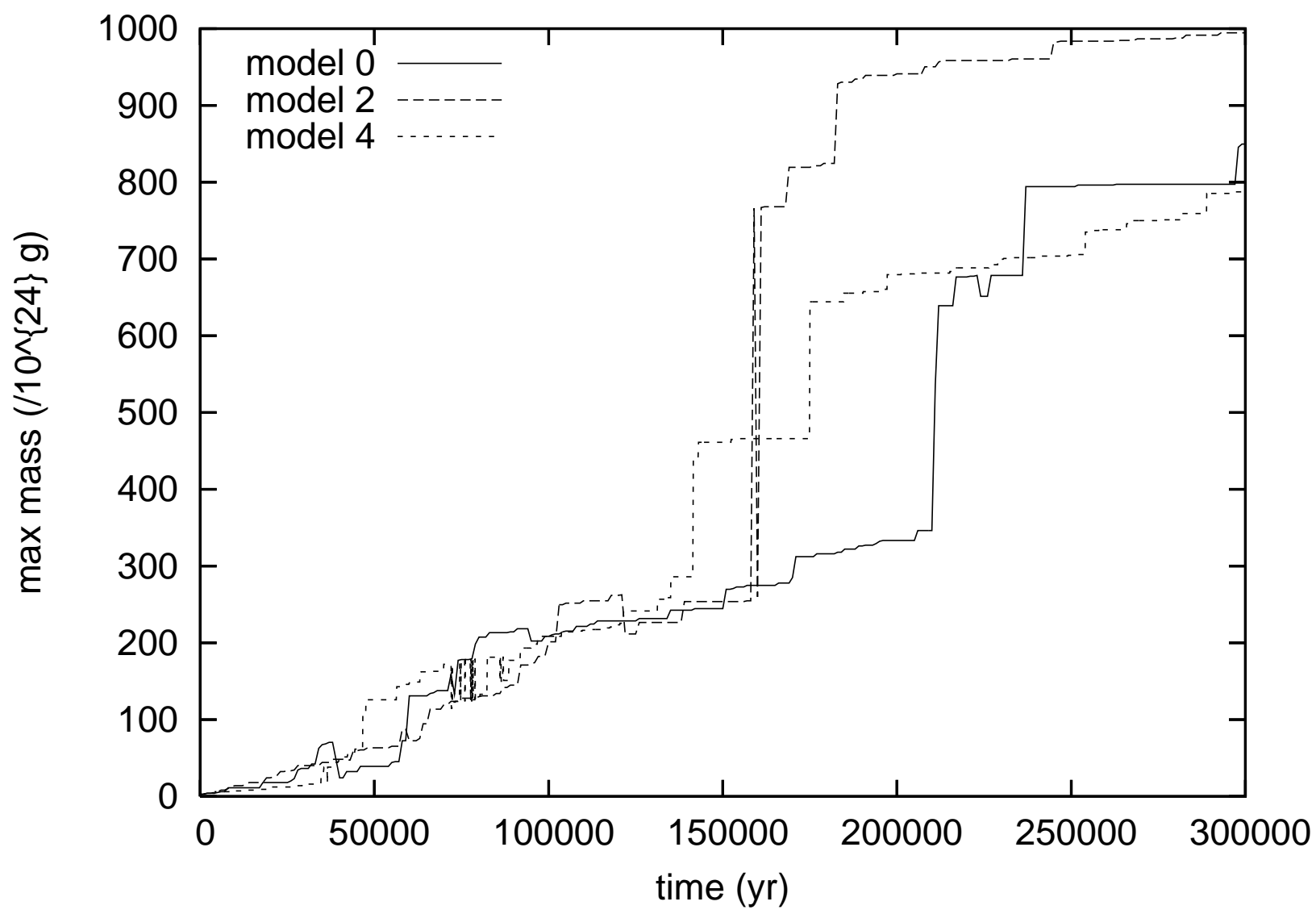

Fig. 4. - Same as figure 3 but for models 0,2 , and 4 . 


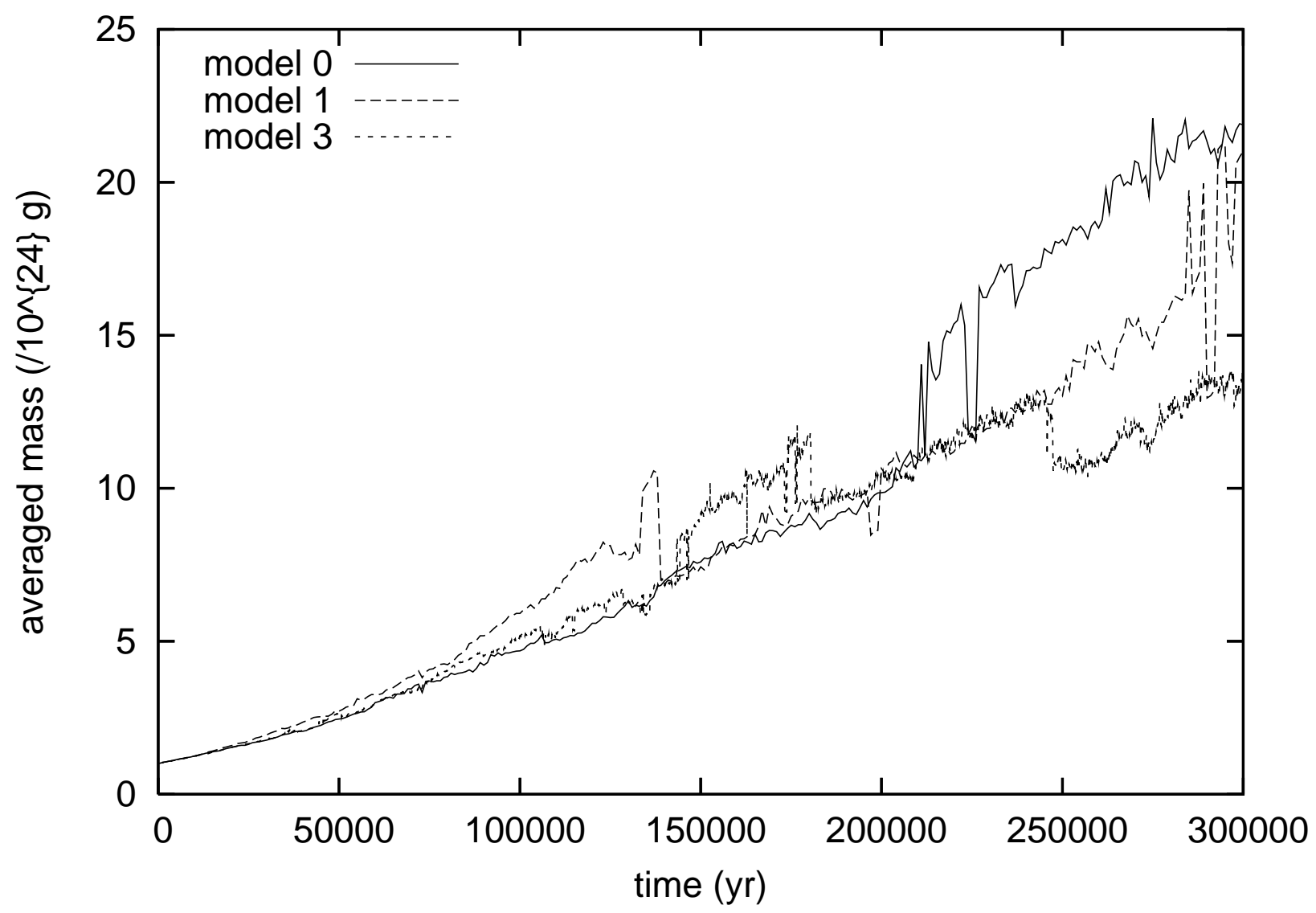

Fig. 5.- Same as figure 3 but the average mass is shown. 


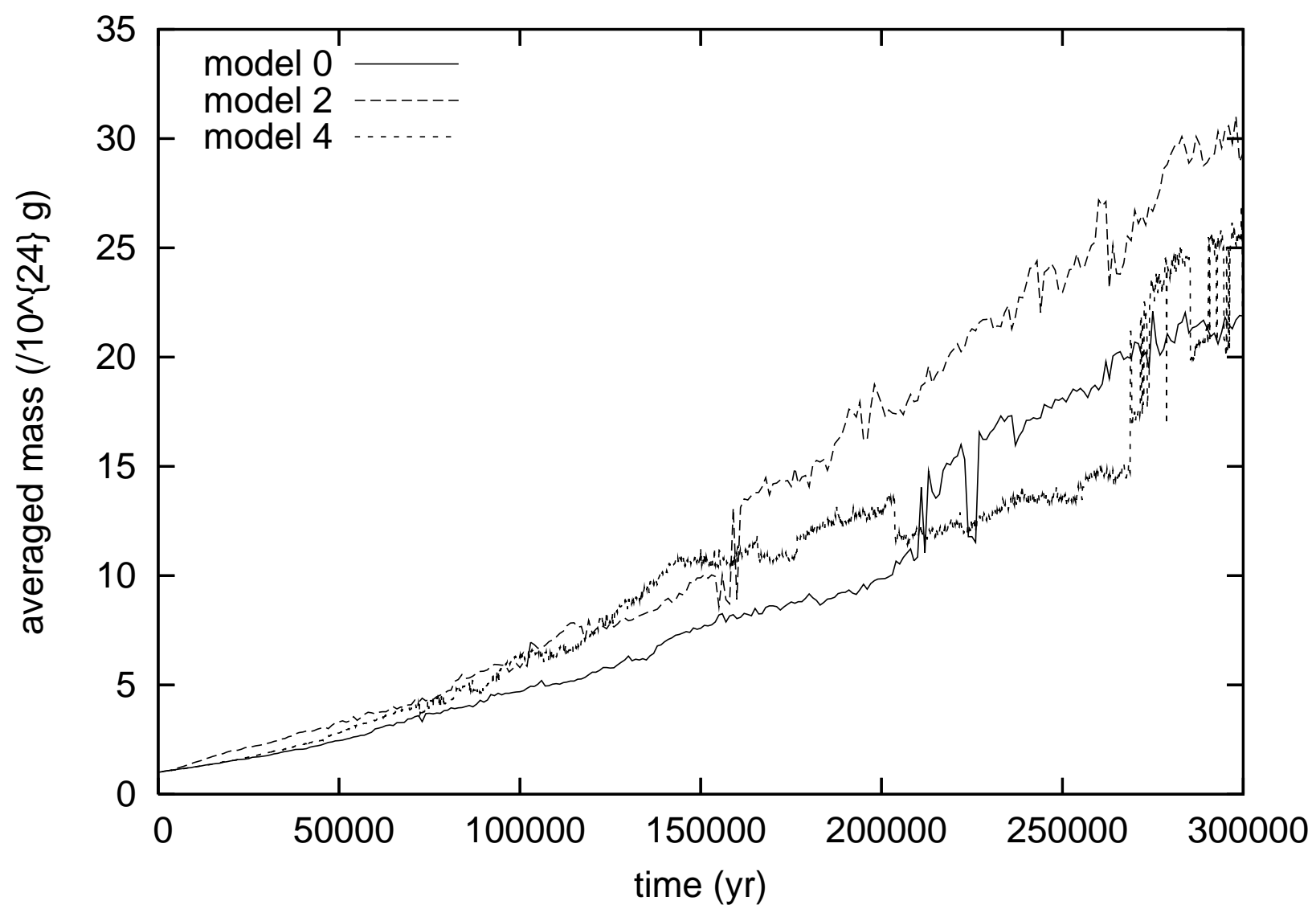

Fig. 6. - Same as figure 5 but for models 0, 2, 4 . 


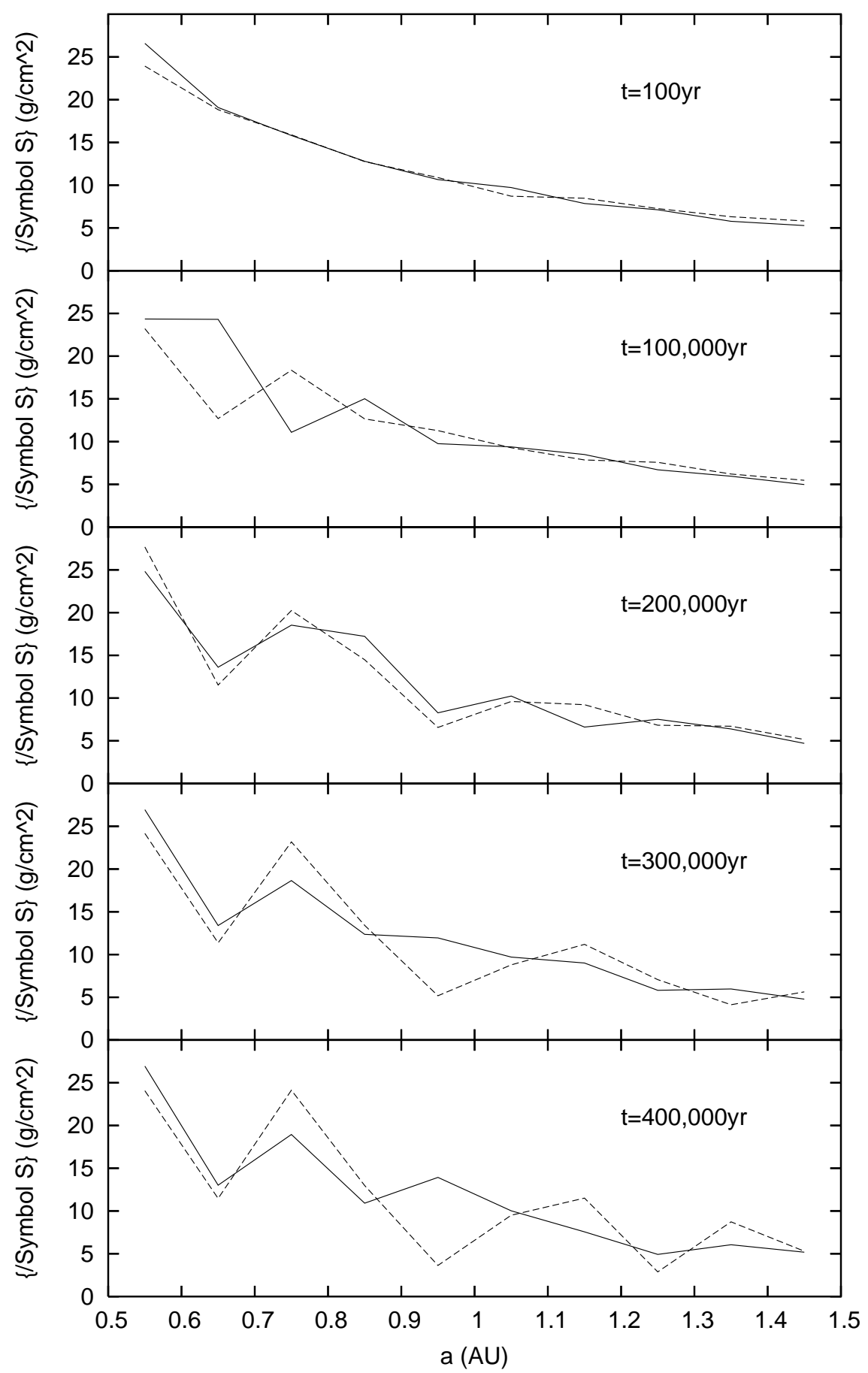

Fig. 7.- The surface mass density of planetesimals for model 1 (solid) and model 0 (dotted) at $t=1 \times 10^{2}, 1 \times 10^{5}, 2 \times 10^{5}, 4 \times 10^{5}$ years, from top to bottom. 


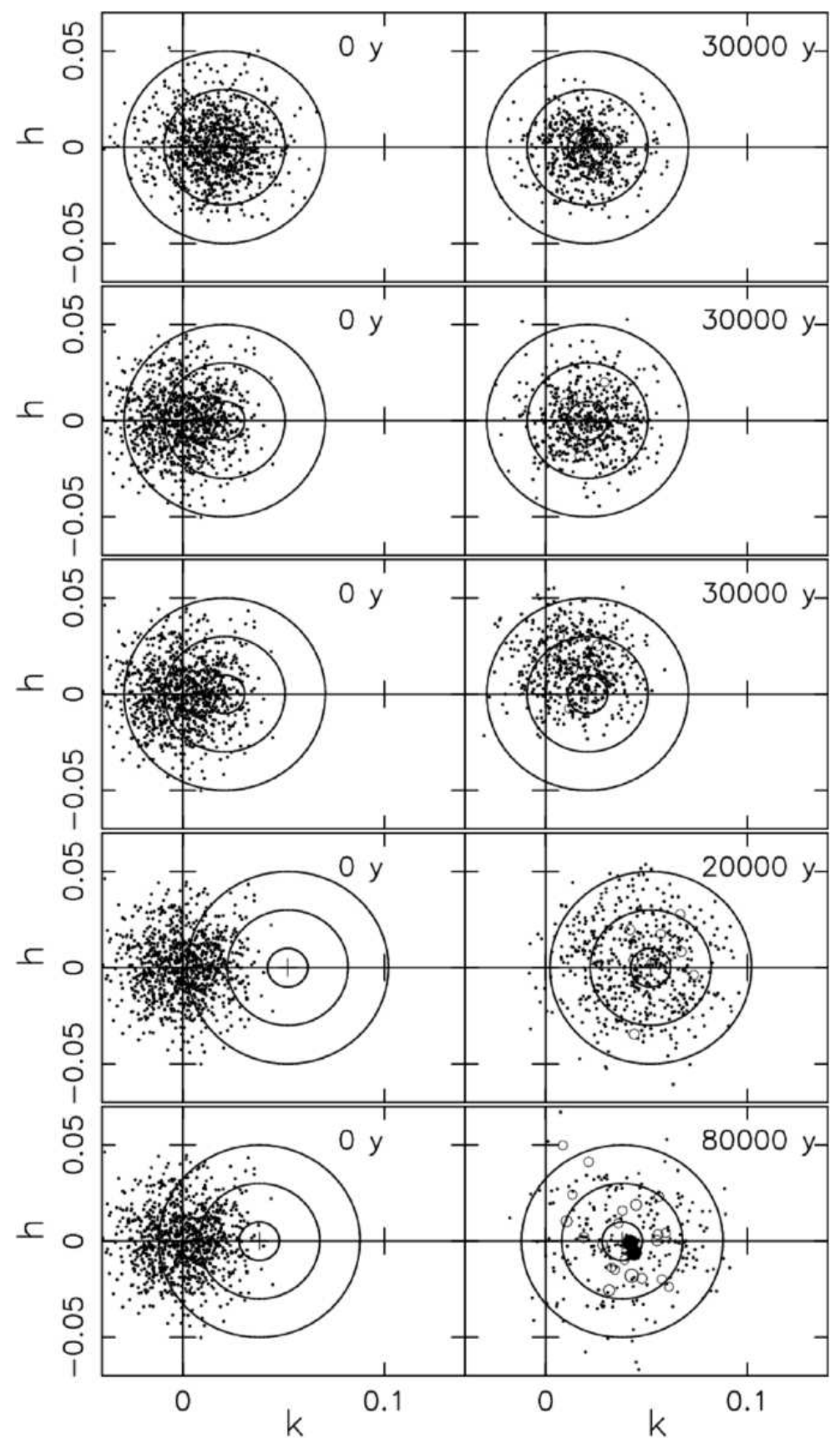

Fig. 8.- The distribution of planetesimals of model 1, 3, 8, 4, 5 (top to bottom) on k-h plane. Left panels show initial distributions and right panels show evolved state $\left(3 \times 10^{4}\right.$ years, for models 1,3 , and 8 and $2 \times 10^{4}$ and $8 \times 10^{4}$ years for models 4 and 5) We plotted planetesimals between $0.95 \mathrm{AU}<a<1.05 \mathrm{AU}$. The center of circles is the forced eccentricity for $a=1 \mathrm{AU}$ and radii of circles are $0.01,0.03,0.05$ respectively. The open and filled circles represent the planetesimals with mass 10 times and 100 times the initial value. 


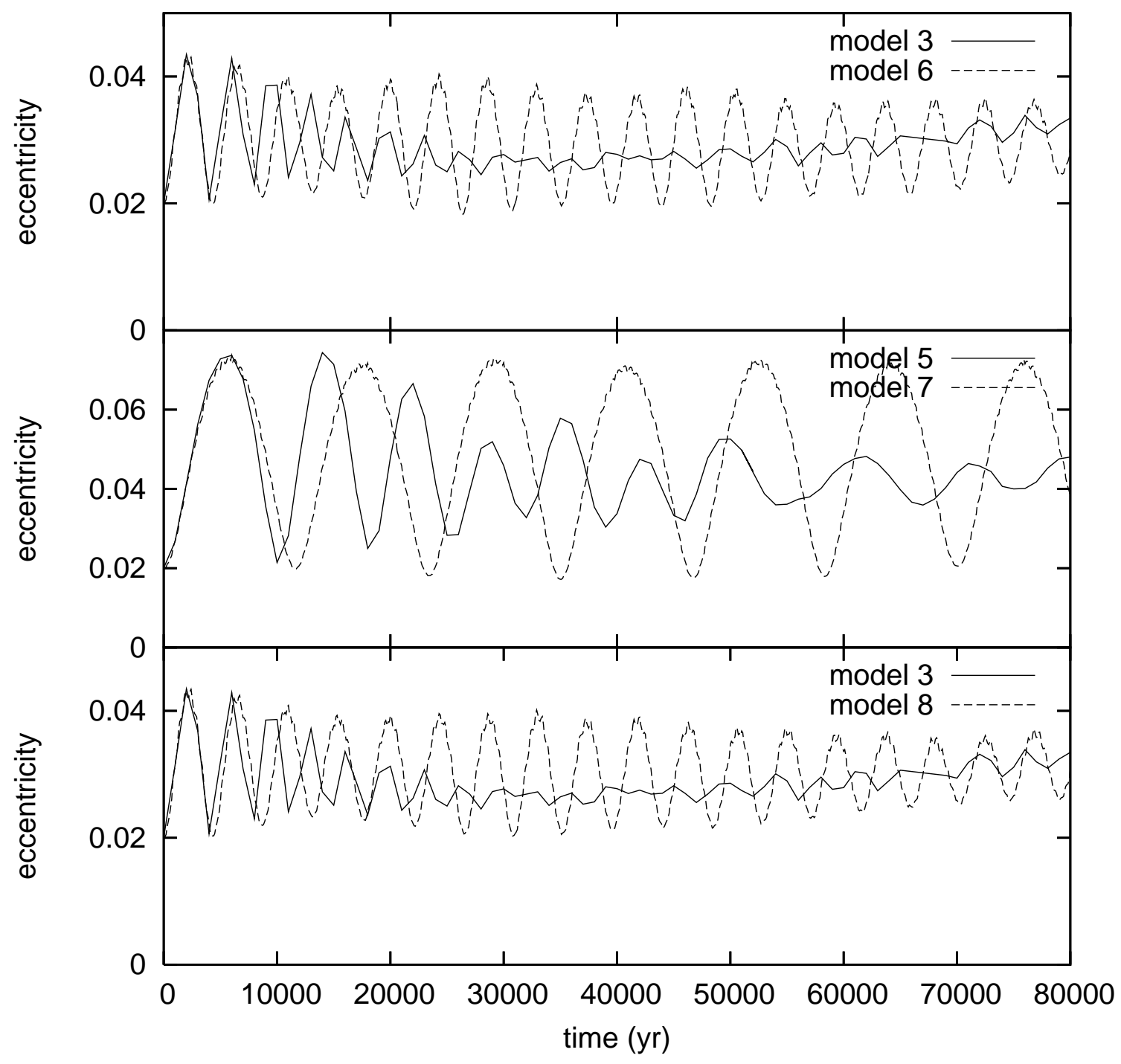

Fig. 9.- The time evolution of the average eccentricity in narrow disk models (models 6 8) compared with those in wide disk models (models 3, 5, and 3 from top to bottom). For wide disk models, the eccentricity is averaged for planetesimals in the range of $0.95 \mathrm{AU}<$ $a<1.05 \mathrm{AU}$. The solid curves show results of wide disk models and dashed curves show results of narrow disk models. 


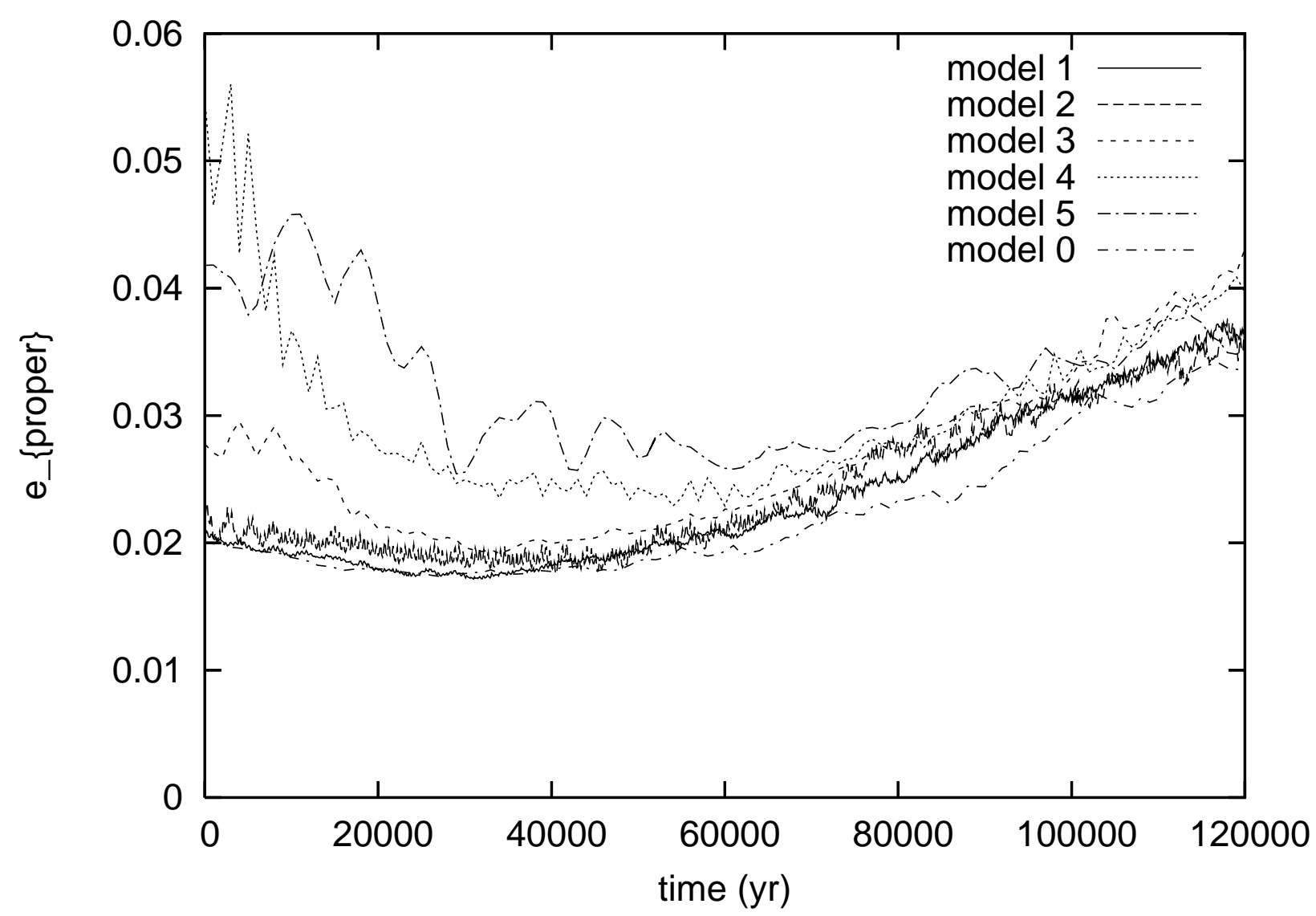

Fig. 10.- The time evolution of average of proper eccentricity. the proper eccentricity is averaged for planetesimals with $0.95 \mathrm{AU}<a<1.05 \mathrm{AU}$. Solid, long-dashed, short-dashed, dotted, and upper dot-dashed curves show the results of models $1-5$, respectively. Lower dot-dashed curve shows the result of model 0 . 


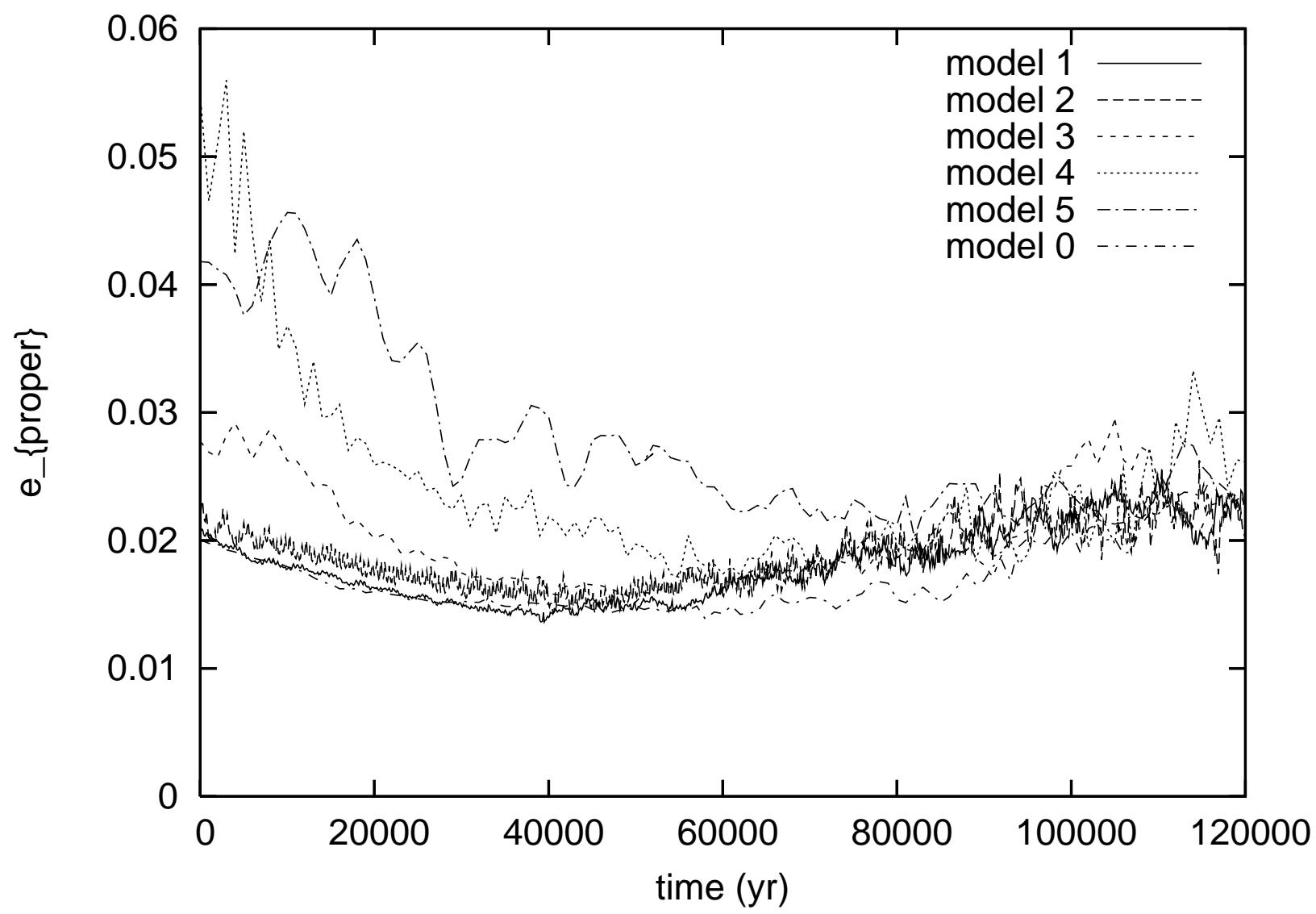

Fig. 11. - The same as figure 10 but mass-weighted averages are shown. 